\title{
STRESS-STRAIN STATE AT FORCE AND TEMPERATURE LOADING OF ASSEMBLES FROM DISSIMILAR STEELS WITH SOFT INTERLAYER
}

\author{
I.A. KOLESAR and G.V. ERMOLAEV \\ Admiral Makarov National Shipbuilding University \\ 9 Geroev Stalingrada Ave., 54025, Nikolaev, Ukraine. E-mail: welding@nuos.edu.ua
}

\begin{abstract}
We have studied the stress-strain state (SSS) at loading by compression and temperature variation, in order to optimize the process of diffusion welding and brazing of assembles from dissimilar materials with soft interlayers. Materials having different thermal coefficient of linear expansion (TCLE) and the same moduli of elasticity were joined. An example of such joints can be those of magnetic and nonmagnetic steels produced with application of diffusion welding with soft interlayers or pressure brazing using filler metals based on copper with silicon, manganese and boron additives. Sufficient strength of materials in the brazed assembly is combined with plastic deformation of just the interlayer. Fields of all the components of stresses and strains, as well as their diagrams in different assembly sections were studied. Analysis of simulation results showed that tangential and equivalent stresses in the butt zone (on the interface), determining formation of physical contact and activation of the process of joint formation in diffusion welding, at simultaneous loading of assemblies with a soft interlayer rise considerably in material with higher TCLE at cooling and in material with lower TCLE at heating. Distribution of plastic deformations in the interlayer is more uniform, i.e. thermal cycling under pressure promotes formation of physical contact and activation of processes of joint formation in such assembles. 8 Ref., 10 Figures.
\end{abstract}

$\boldsymbol{K} \boldsymbol{e} \boldsymbol{y} \boldsymbol{w} \boldsymbol{o} \boldsymbol{d} \boldsymbol{s}:$ diffusion welding, brazing, dissimilar materials, soft interlayers, SSS simulation, temperature and force loading

Diffusion welding (DW) and brazing are the main methods to join many materials, which cannot be joined by fusion welding in connection with the loss of their structure and properties.

One of the important problems of joining dissimilar materials by these methods is plastic deformation and activation of the stronger material surface. The existing problems are solved through application of DW with intermediate interlayers and with controlled stress-strain state (SSS) [1]. In the case of application of brazing, the braze metal proper often is the intermediate interlayer, which can have different physico-mechanical properties both before brazing, and after interaction with the base material.

Over the recent years a lot of attention has been given to studying the SSS in DW and brazing of assembles from dissimilar materials [2-7], that is why investigation of the influence of strength ratios of materials being joined and the interlayer on SSS of the assembles and establishing the general regularities is urgent.

The objective of this work is determination of the influence of low strength of material of intermediate interlayers, compared to that of materials being joined (soft interlayers) and SSS

(C) I.A. KOLESAR and G.V. ERMOLAEV, 2014 formation both during DW and during cooling after welding and brazing.

Investigations were conducted by computer simulation method with application of licence ANSYS software (version 10). Axisymmetric problems were solved with application of finite elements (FE) of PLANE 182 type. Adequacy of simulation results was tested experimentally [8].

Assembly types most often encountered at DW and brazing were studied, namely bushingbushing $(\mathrm{B}-\mathrm{B})$ and cylinder-cylinder $(\mathrm{C}-\mathrm{C})$ type. The latter type of assembly can be regarded as a particular case of the first one, when inner diameter is turned to zero. General view of physical and FE models is shown in Figure 1. Interlayer thickness was taken to be equal to $1 \mathrm{~mm}$, total height of all the assembles $2 h-20 \mathrm{~mm}$, cylinder radius and bushing thickness $-10 \mathrm{~mm}$.

Investigations were performed on assembles from materials of the same rigidity (modulus of elasticity $E=2 \cdot 10^{5} \mathrm{MPa}$ ) and strength (yield point $\sigma_{y} \geq 200 \mathrm{MPa}$ ) with interlayers of the same rigidity, but lower strength than that of base materials $\left(\sigma_{\mathrm{y}}=38 \mathrm{MPa}\right)$. For material 1 thermal coefficient of linear expansion (TCLE) $\alpha \cdot 10^{6}=$ $=20 \mathrm{deg}^{-1}$; for material $2-10$; for interlayer 15. Material phisico-mechanical properties and load level were selected so that only interlayer material was plastically deformed, and deformation affected the greater part of interlayer.

Force (compression up to $40 \mathrm{MPa}$ ), temperature (cooling by $100{ }^{\circ} \mathrm{C}$, when SSS is induced due to 

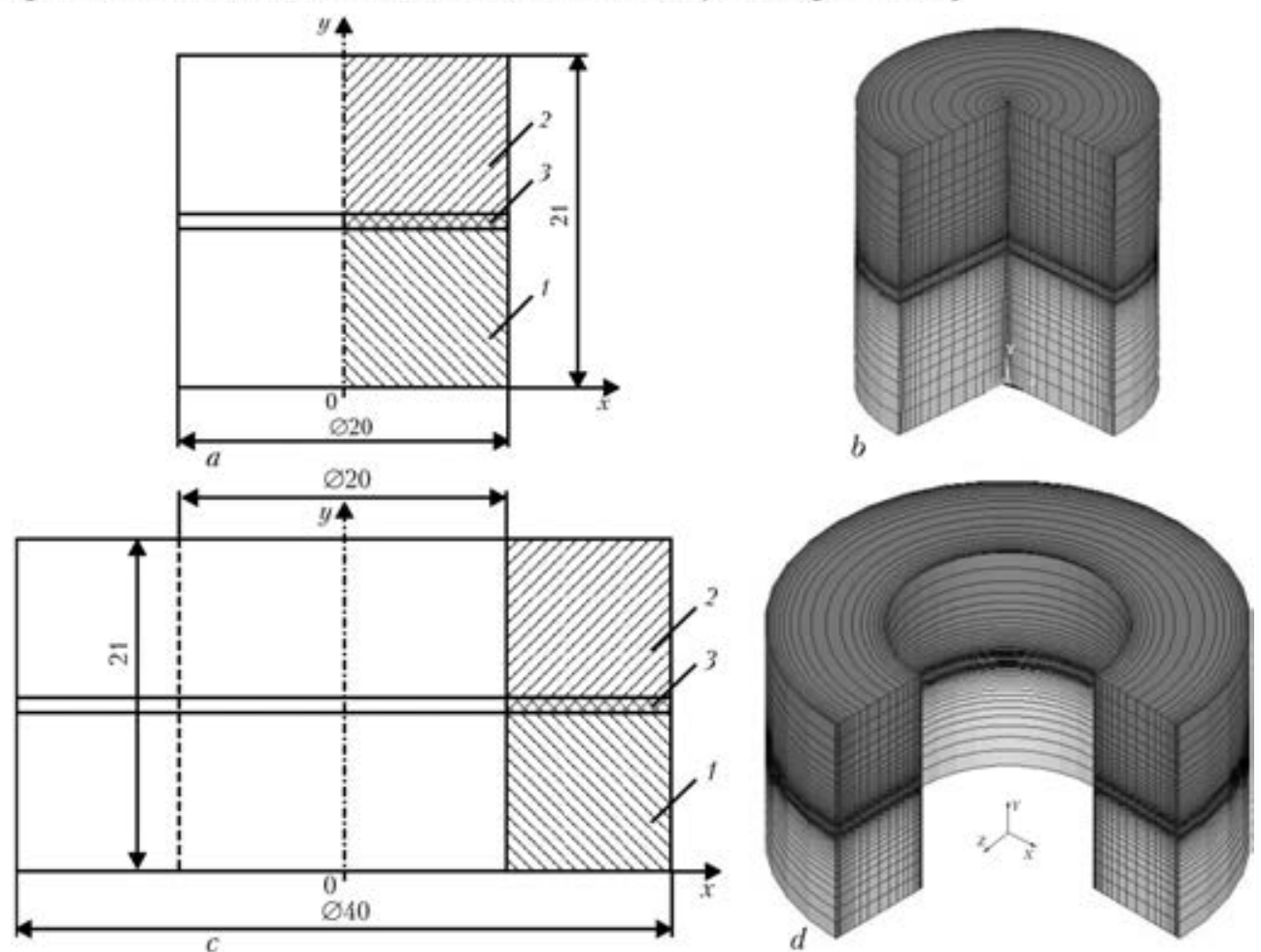

Figure 1. Physical $(a, c)$ and FE $(b, d)$ models of assemblies of $\mathrm{C}-\mathrm{C}(a, b)$ and $\mathrm{B}-\mathrm{B}(c, d)$ type with an interlayer: 1 , 2 - materials 1 and 2 , respectively; 3 - interlayer

difference in TCLE of materials being joined) and simultaneous temperature-force loading of the assembly (after joint formation) was considered. It is obvious that results obtained at such a loading are found also when cooling is replaced by heating (in welding with thermal cycling), but materials 1 and 2, having different TCLE, will change places in this case. Simulation results were compared with those for similar assembles at different kinds of loading and with each other.

Fields and diagrams of all the components of stresses and plastic deformations of assemblies were analyzed. As shown by analysis of results of simulation in $\mathrm{C}-\mathrm{C}$ and $\mathrm{B}-\mathrm{B}$ assemblies, the nature of SSS on the whole corresponds to general principles of mechanics and earlier established regularities [2, 5-7]. Here, SSS of $\mathrm{C}-\mathrm{C}$ and $\mathrm{B}-\mathrm{B}$ assemblies differs only near the bushing inner surface, which is absent in $\mathrm{C}-\mathrm{C}$ assemblies. Therefore, Figures 2-4 give only the fields in B-B assembles.

In the interlayer region a bulk stressed state is induced, which is the most clearly expressed at temperature and simultaneous temperatureforce loading. At purely force loading, the stressed state changes only slightly, remaining close to the linear one.

In assemblies of both types, the effects of temperature and force loading are summed up algebraically, as a result of which the fields of radial and circumferential stresses remain practically the same, as at purely temperature loading. Axial
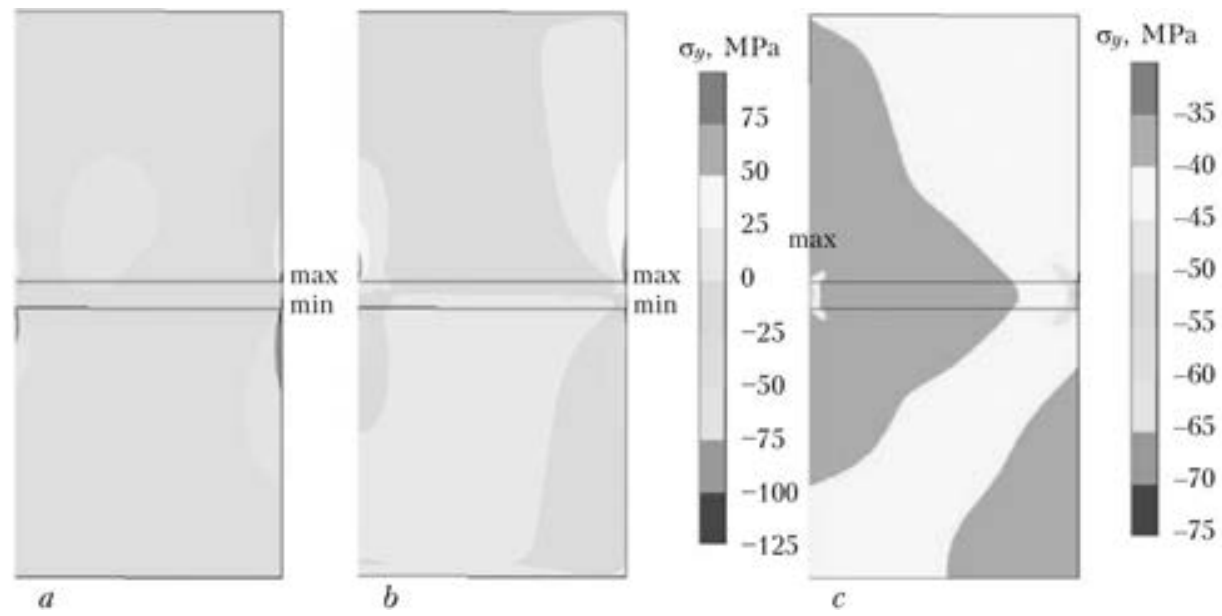

Figure 2. Fields of axial stresses in assemblies B-B with soft interlayer at temperature-force $(a)$, temperature $(b)$ and force $(c)$ loading 

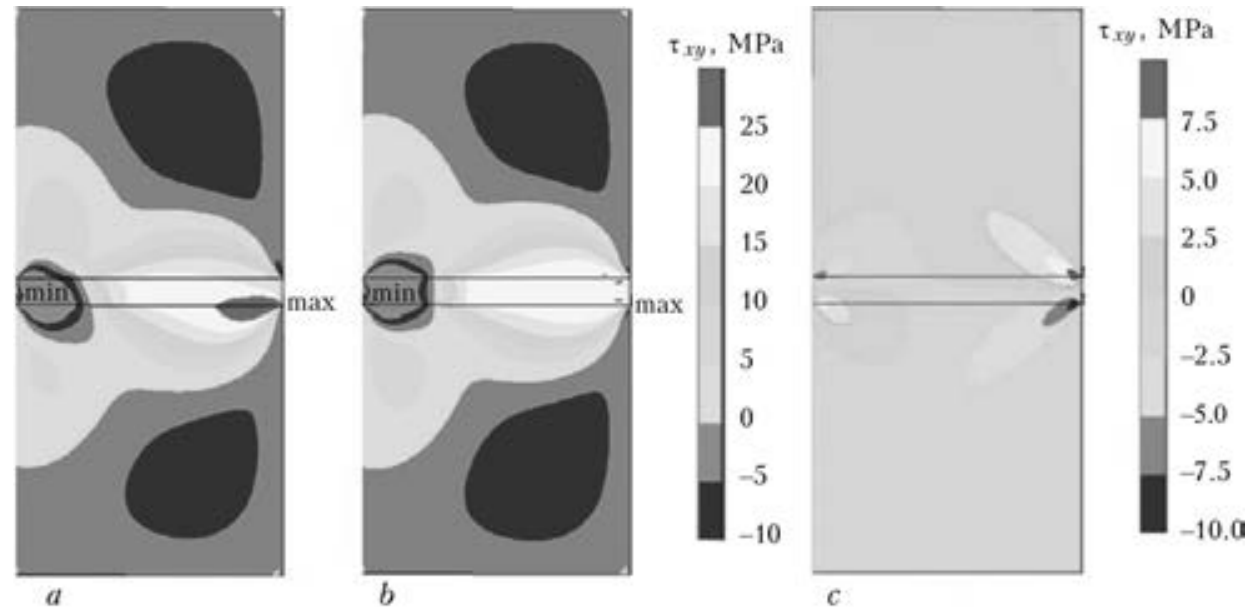

Figure 3. Fields of tangential stresses in assemblies B-B with soft interlayer at temperature-force $(a)$, temperature $(b)$ and force $(c)$ loading

compressive stresses in material 1 increase, and tensile stresses in material 2 decrease by the magnitude of compressive load (see Figure 2).

Tangential stresses are noticeably increased on the interface of the soft interlayer and material 1 and decrease on the interface with material 2 (see Figure 3), compared to purely temperature loading. Here, algebraic summation of effects due to the difference of TCLE and plastic deformation of the interlayer is clearly manifested.

Field of equivalent stresses changes in a similar fashion (see Figure 4). The latter noticeably rise in material 1 and decrease in material 2 at simultaneous loading compared to purely temperature loading.

The field of plastic deformations also changes in keeping with equivalent stresses (Figure 5). Similar to purely temperature loading, the maximum of deformations is concentrated near the assembly outer surface, but their distribution across the interlayer thickness changes markedly. They are maximum at the interface with material 1 and decrease with increasing distance from it.

Shape of diagrams of distribution of radial, circumferential and tangential stresses in the ma- terials being joined at simultaneous temperature and force loading coincides with the shape of the respective diagrams at purely temperature loading. Maximum radial stresses in materials being joined decrease by 15-20 $\mathrm{MPa}$, and circumferential stresses - by 10-15 MPa. Diagrams of axial stresses shift towards compression by $40 \mathrm{MPa}$ (Figure 6).

Maximum tangential stresses near the outer cylindrical surface increase from the side of material 1 by $10 \mathrm{MPa}$ (Figure 7, $a$ ) and decrease by $10 \mathrm{MPa}$ from the side of material 2 (Figure $7, b$ ).

Diagrams of equivalent stresses change accordingly. The level of these stresses increases by the magnitude of applied pressure of $40 \mathrm{MPa}$ in material 1 and decreases by $40 \mathrm{MPa}$ in material 2 (Figure 8). Their distribution in both the materials being joined remains close to the uniform one.

In the soft interlayer material, which is plastically deformed, the magnitude of equivalent stresses remains on the level of about $40 \mathrm{MPa}$ from the side of material 2 on the greater part of the joint. From the side of material 1 the distribution is nonuniform with clearly outlined stagnation zones (near axis of assemblies $\mathrm{C}-\mathrm{C}$
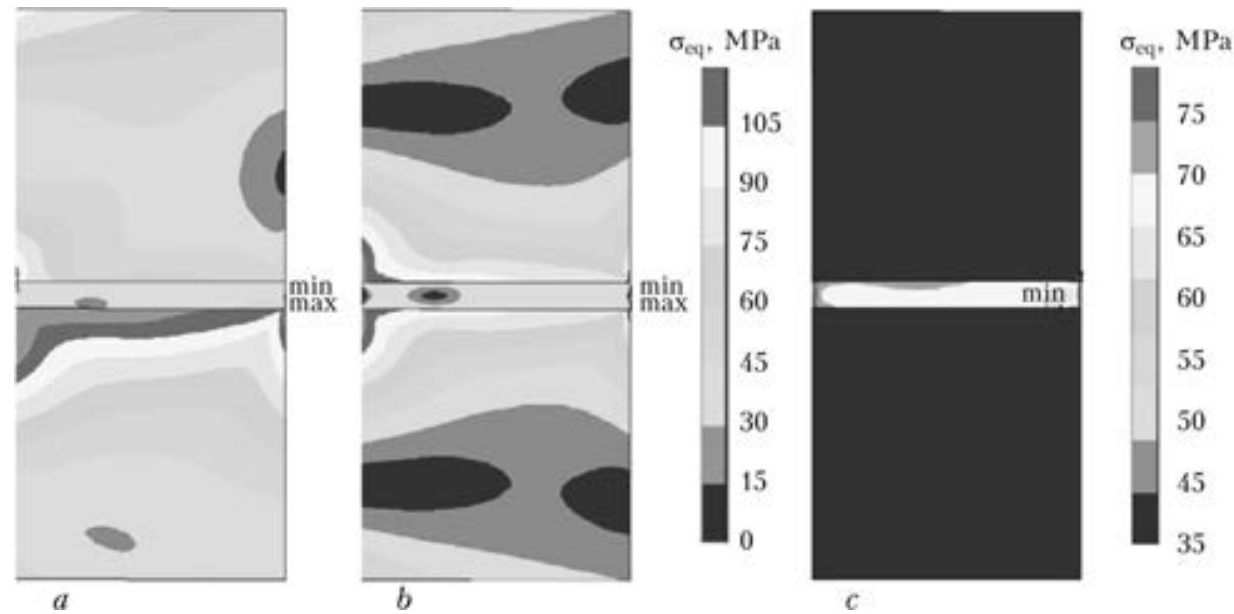

Figure 4. Fields of equivalent stresses in assemblies B-B with soft interlayer at temperature-force $(a)$, temperature $(b)$ and force $(c)$ loading 
Welding Production Chair of Admiral Makarov National Shipbuilding University

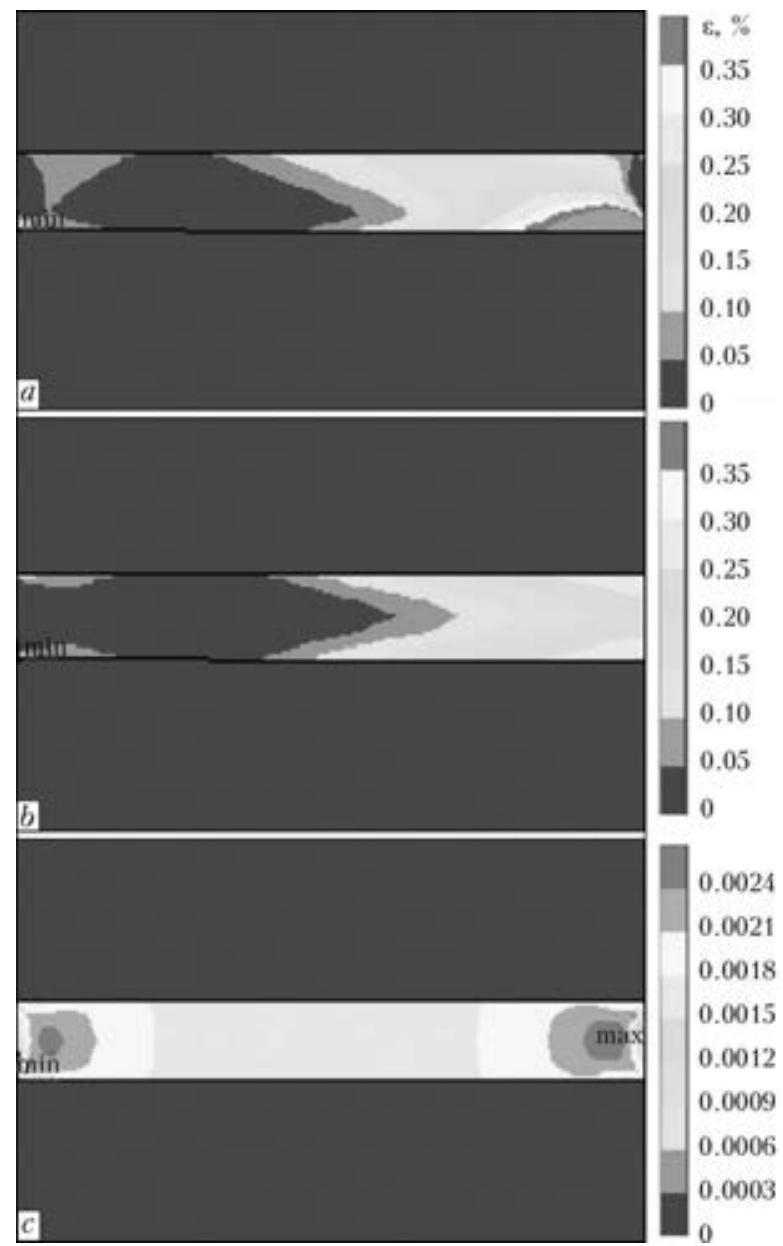

Figure 5. Fields of plastic deformations in soft interlayer of assemblies B-B at temperature-force (1), temperature (2) and force (3) loading

and at $1 / 3$ of thickness from inner side of assemblies B-B), in which equivalent stresses drop to 10-25 MPa. Near the assembly outer surface they, contrarily, rise owing to work hardening at high plastic deformations.
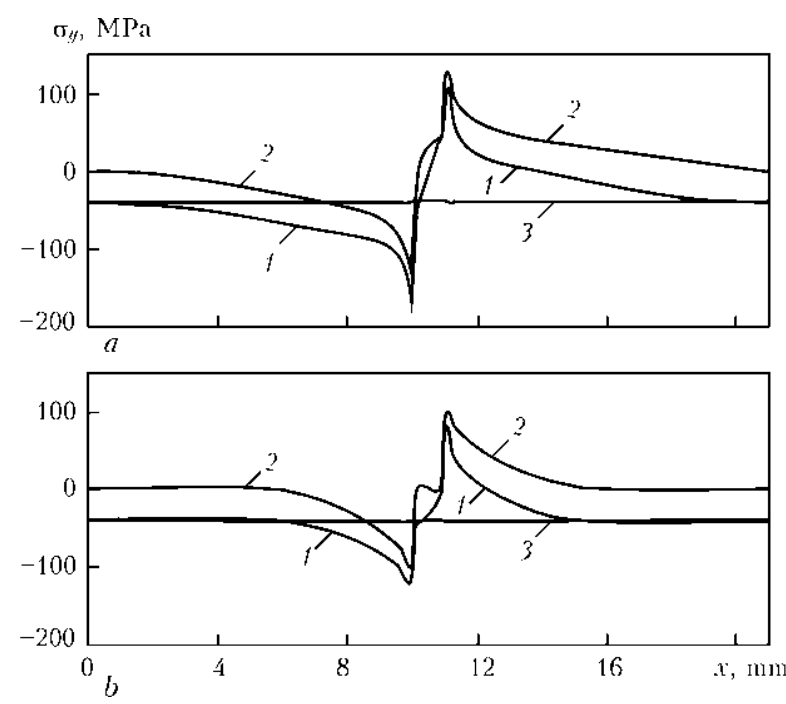

Figure 6. Axial stress diagrams along outer $(a)$ and inner (b) surfaces of assembly B-B with soft interlayer at temperature-force (1), temperature (2) and force (3) loading
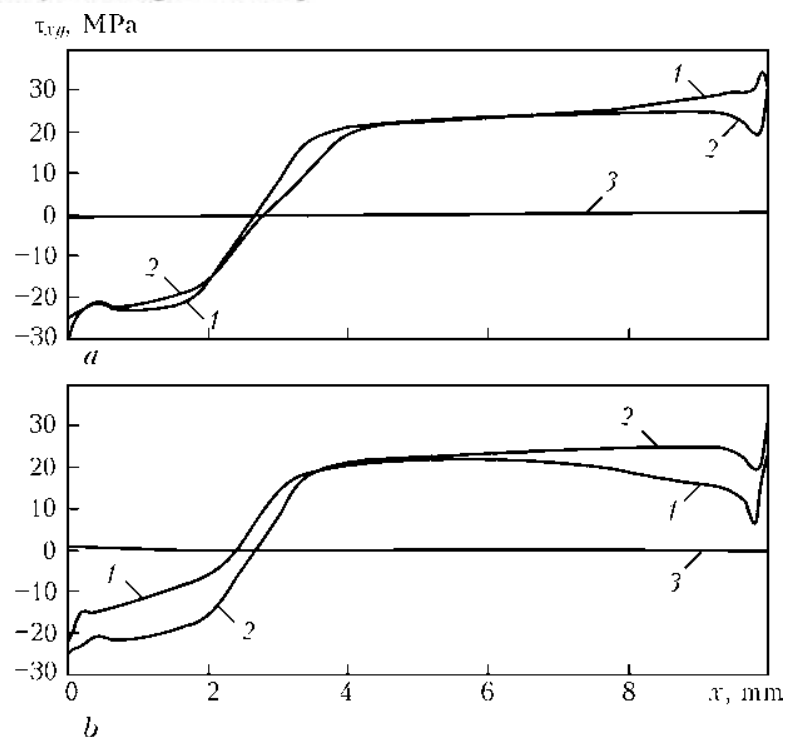

Figure 7. Diagrams of tangential stresses along the interfaces of materials $1(a)$ and $2(b)$ being joined with interlayer in assemblies B-B at temperature-force (1), temperature (2) and force (3) loading

Analysis of plastic deformation diagrams in the soft interlayer material also shows that they are distributed nonuniformly, rising gradually from those close to 0 in the stagnation zone up to $1 \%$ and more near the outer surface. Here, on the boundary with material 1 in this zone they are several times higher at simultaneous loading than at purely temperature loading (Figure 9, $a$ ). On the boundary with material 2 , contrarily, their distribution is more uniform than at purely temperature loading (Figure 9, b), but their level is lower.

Thus, simultaneous force (compression) and temperature (cooling) loading creates more favourable conditions for inducing plastic deformations in the soft interlayer than the purely thermal loading does. From the side of material 1 (with higher
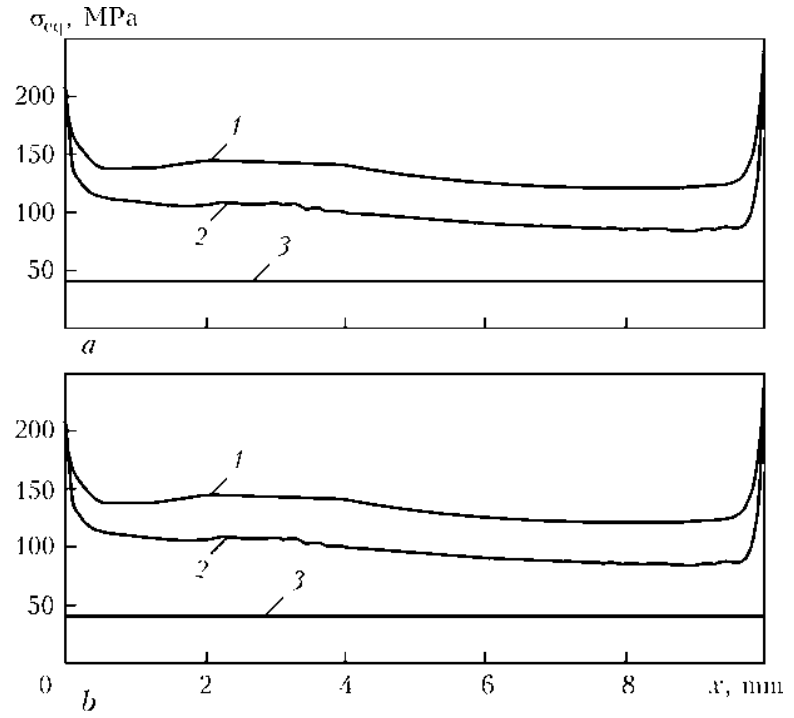

Figure 8. Diagrams of equivalent stresses in materials 1 and 2 being joined along the interfaces with interlayer in assemblies B-B at temperature-force (1), temperature (2) and force (3) loading 

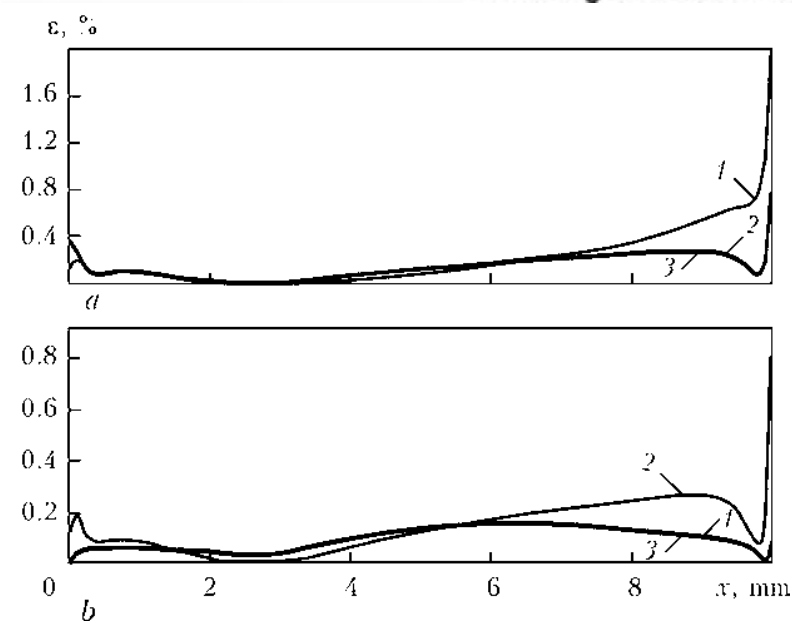

Figure 9. Diagrams of plastic deformations in interlayer material at the interface with materials $1(a)$ and $2(b)$ being joined in assemblies B-B at temperature-force (1), temperature (2) and force (3) loading

TCLE) their level and distribution nonuniformity rise, from the side of material 2 (with lower TCLE) they decrease, but their distribution becomes more uniform. It is obvious that when cooling is replaced by heating, the materials change places, i.e. thermal cycling under pressure should promote formation of physical contact and activation of the processes of joint formation.

For convenience of comparison, Figure 10 gives diagrams of stresses maximum by their modulus in the materials being joined and the interlayer at different loading variants.

Diagram analysis confirms that the effect of simultaneous temperature and force loading of assemblies with soft interlayers is manifested in a certain increase of radial and tangential stresses in all the materials, increase of equivalent stresses in material 1 and the interlayer and of axial stresses in materials being joined, and lowering of equivalent stresses in material 2 and axial stresses in the interlayer material, respectively, tangential stresses remaining practically the same as at purely temperature loading.

\section{Conclusions}

1. Tangential and equivalent stresses in the butt zone (on the interface), determining formation of physical contact and activation of the process of joint formation in DW, at simultaneous loading of assembles with soft interlayer noticeably increase in the material with higher TCLE values at cooling and in material with lower TCLE at heating. Distribution of equivalent stresses is close to the uniform one, i.e. thermal cycling under pressure should promote formation of physical contact and activation of the processes of joint formation in assemblies with a soft interlayer.

2. Plastic deformations in material of soft interlayer on the interface with material with lower
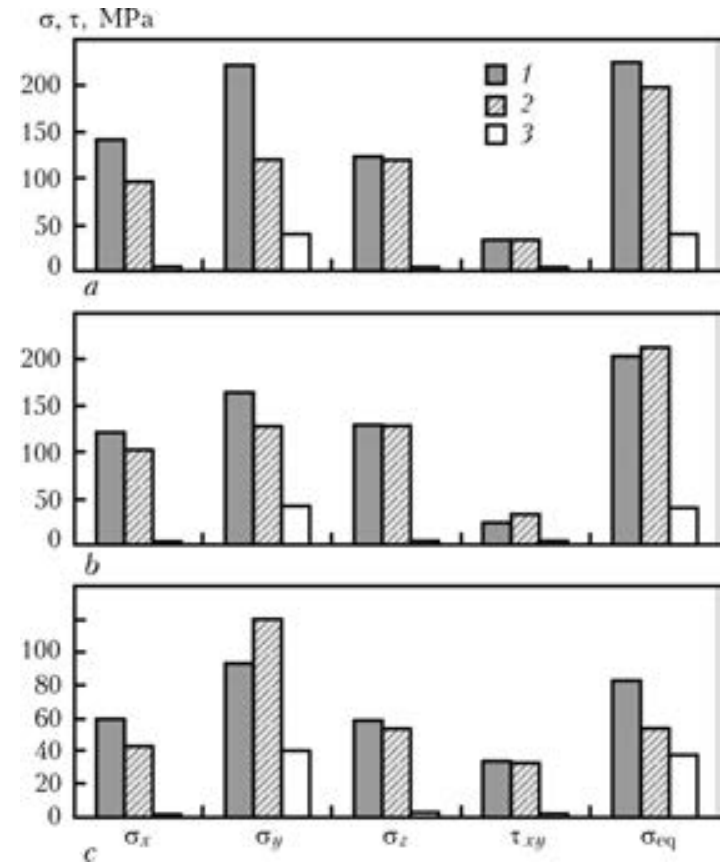

Figure 10. Maximum (by modulus) stresses in materials 1 $(a), 2(b)$ and soft interlayer (c) of assemblies B-B at temperature-force (1), temperature (2) and force (3) loading

TCLE at simultaneous loading by compression and cooling are distributed more uniformly, but their level is lower than at purely temperature loading. At simultaneous compression and heating the same takes place on the interface with material with higher TCLE, i.e. welding with thermal cycling under pressure in assembles with a soft interlayer provides a more uniform distribution of plastic deformations in the interlayer.

1. Kvasnitsky, V.V., Matvienko, M.V., Ermolaev, G.V. et al. Method of diffusion welding of materials. Pat. 81583 Ukraine. Int. Cl. B23K 20/14. Fil. 21.11.2006. Publ. 10.01.2008.

2. Kvasnitsky, V.V., Ermolaev, G.V., Matvienko, M.V. (2007) Principles of formation of stress-strain state in diffusion welding of dissimilar materials for cylindercylinder and bush-bush assemblies. Zbirnyk Nauk. Prats NUK, 5, 57-65.

3. Zhong, Z., Zhou, Z., Ge, C. (2004) Residual stress distribution and effect of interlayer in doped graphite / copper joints. Welding Technology, 6, 14-16.

4. Zhong, Z., Zhou, Z., Ge, C. (2009) Brazing of doped graphite to $\mathrm{Cu}$ using stress relief interlayers. J. $\mathrm{Ma}$ terials Proc. Techn., 209, 2662-2670.

5. Makhnenko, V.I., Kvasnitsky, V.V. (2009) Stressstrain state of assemblies of the cylindrical shape in diffusion bonding. The Paton Welding J., 2, 2-7.

6. Makhnenko, V.I., Kvasnitsky, V.V. (2009) Peculiarities of formation of stress-strain state in diffusion bonds between dissimilar materials. Ibid., 8, 7-11.

7. Kvasnitsky, V.V., Ermolaev, G.V., Kolesar, I.A. (2011) Stress-strain state of welded and brazed joints of same rigidity dissimilar materials with interlayers. Zbirnyk Nauk. Prats NUK, 5, 36-43.

8. Kvasnitsky, V.V., Zolotoj, Yu.G., Labartkava, A.V. et al. (2008) Experimental investigation of deformation of welded joint of bush-bush type from dissimilar materials. Ibid., 4, 65-73. 